\title{
1 Introduction: Ceramic Archaeometry and Paste Analysis
}

Paste analysis is essential in the study of archaeological ceramics. These analyses can be performed through different strategies that have diverse scale, accuracy, precision, complexity and sophistication. We can roughly distinguish between macroscopic and microscopic or archaeometric analysis. Although in practice both types of approaches are usually combined, this book is basically centred on the archaeometric study of ceramic pastes and fabrics. That is why it is appropriate to start this chapter conducting a brief evaluation of the basic fundaments of Archaeometry and its current role in the investigation of past societies.

Archaeometric studies of pastes and fabrics are fundamental to the classification and characterization of pottery, providing relevant data, among other aspects, about its production, function and social meaning. Several authors (e.g., Jones, 2004; Maniatis, 2002; Montero et al., 2007; Pollard and Heron, 2008; Vidal, 2008a) have already summarized the background of archaeometry and developed an overview of its role in archaeology. The fact is that archaeometric studies, especially in archaeological ceramics, have developed significantly since the 1960 s (e.g., Peacock, 1970; Sheppard, 1971; Tite, 1969). This significant advance, at least in Europe and North America, has enhanced the analytical study of archaeological materials and the topics considered in the study of material culture in recent decades.

Archaeometry encompasses a group of analytical techniques applied in the study of material culture with the aim to obtain a quantitatively and qualitatively rich and diverse corpus of data. The data collected with these techniques provide relevant information concerning the ceramic technology of past societies that eventually permits us to approach the way these societies conceptualized, produced, used, maintained, exchanged and deposited their pottery. Furthermore, archaeometry is based on the necessary interdisciplinary relationship between diverse branches of the natural and social sciences. This relationship is essential in archaeology, since, from physical materials (objects), scholars have to face questions that go beyond the limits of the tangible and pertain instead to abstract and social concerns. In short, with the application of techniques and methods from the natural sciences such as physics, chemistry, geology and micropaleontology it aims at dealing with the enquiries associated with human and social sciences.

As pointed out by Montero et al., (2007), the use of archaeometry allows the establishment of new viewpoints regarding the analysis of material culture. Although methodology is always embedded in particular theoretical trends, we have to consider that the application of archaeometric techniques provides a diverse and comprehensive framework of data that is useful for studying technology through multiple interpretative frameworks. Thus, the characterization of the ceramic's technological features through archaeometric techniques permits us to collect a 
broad and solid dataset from which we can develop different interpretations about the society that created and/or used the pottery.

Therefore, studies focused on archaeological ceramics have been substantially enriched by the implementation of chemical, physical and mineralogical analyses. This improvement has enabled us to transcend the analytical scales and the information that used to be obtained by less sophisticated macroscopic methods or the typological classification of the vessels. Thus, the use of archaeometric analytical techniques has allowed us to increase the data available concerning the material culture of past societies (Cordero et al., 2006; Jones, 2002). The advantages of ceramic archaeometry for addressing many archaeological enquiries resulted, especially since the 1980s, in the consolidation and continued improvement of research methodologies based on physicochemical analysis. At the same time, we have witnessed the emergence of new analytical techniques, which allow a more accurate approach to the manufacturing techniques and the composition of the artefacts.

Moreover, most archaeometric techniques provide the possibility to approach the materials both in qualitative and quantitative terms. This ability encouraged, in addition to the application of these scientific and technical advances, the emergence of statistical analysis and regulated descriptions in the study of materials (Vidal, 2008a). The quantitative nature of archaeometry, especially when linked with statistics, allows establishing the degree of variability of the pottery features with greater efficiency. Hence, archaeometry and statistical analysis are complementary and provide an operational method to face aspects such as the technological change or the organization of production.

However, the use of archeometric analyses does not necessarily involve deeper interpretations of material culture or further knowledge regarding past societies. In this way, a solid theoretical framework (of which archaeometry is a methodological tool) is an absolutely indispensable starting point to properly approach past societies. In consequence, the archaeometric characterization of pottery technology must start from a number of basic assumptions related to specific archaeological and historical concerns. Therefore, the research should start by setting the theoretical framework that is going to determine the methodology and, particularly in ceramic archaeometry, the combination of several analytical techniques used to collect data (Jones, 2002, 2004).

However, it cannot be forgotten that any explanation of the past should based on the thorough study of the archaeological artefacts, their manufacturing processes and the analysis of the contexts of their production and use. This phase of the research is absolutely necessary, since the archaeological background is, in its essence, material. It is at this level of analysis where archaeometry can play a decisive role. In practice, archaeometry is an analytical option that potentially allows obtaining large amounts of information from each sample tested. The sheer volume of information provided makes it possible to deal not only with a diversity of phenomena but also with the complexity embedded in material culture. 
This book focuses on the study of pottery production and most of the issues that it entails basically through the archaeometric analysis of ceramic pastes and fabrics. The archaeometric characterization of fabrics mainly focuses on defining the petrological, mineralogical, chemical and textural composition of the vessels. In this sense, compositional analyses which can be performed with a huge variety of techniques and methods are of paramount importance to address the characteristics of the ceramic objects. Thus, in this first section the main types of studies and methods commonly used in archaeology to accomplish the analysis of ceramic materials and the range of variables that are usually recorded will be exposed. Also, the archaeological significance that each method has in the study of the ceramic record will be highlighted.

Therefore, we will not approach the many analytical techniques that are associated with each of these compositional analyses and their basic scientific and technical foundations. This greatly exceeds the goals and expectations of this book. Moreover, this information has already been repeatedly collected in several monographs on ceramics (e.g., Cuomo Di Caprio, 1985; Orton et al., 1993; Rice, 1987; Sheppard, 1971; Velde and Druc, 1999) or in volumes addressing analytical methods in archaeology (e.g., Banning, 2005; Esparza and Cárdenas, 2005; Hurcombe, 2007a; Sutton and Arkush, 2002). In any case, some works of a technical nature that must be consulted in order to deal with each type of archaeometric analysis that may be applied to ceramics will be pointed out.

Finally, we must consider the complementarity existing between the different types of studies, methods and techniques commonly applied in the pottery analysis (e.g., typological and archaeometric analysis, see Chapter 3). This combination is advisable, since it implies the application of a more rigorous and complex scientific approach that combines several scales of analysis (Jones, 2002). No technique or method accomplishes by itself all the requirements needed to properly study material culture. The information obtained with a single technique is limited and insufficient to approximate all the technological aspects of ceramics. The study of pottery should be multidisciplinary, so that the conjunction of different methods and analytical perspectives (e.g., ethnoarchaeology and experimentation with archaeological studies, or combining chemical and petrographic analyses) provide a greater number of findings that makes our inferences about material culture more significant. Consequently, most researchers currently complement the potential of chemical, mineralogical and petrographic studies with other analytical approaches. The combination of several methods provides consistent information regarding ceramic technology that increases the knowledge potentially available to address the dynamics involving the production, use and exchange processes (Tite, 2008). 